\title{
SÉROPRÉVALENCE POUR LA LEUCOSE ENZOTIQUE BOVINE DANS LE CHEPTEL LAITIER DE 'SERTÃO’ DE L'ETAT D'ALAGOAS, NORD-EST DU BRÉSIL
}

\author{
Francisco Feliciano da SILVA JUNIOR ${ }^{\mathbf{1}}$; José Andreey Almeida TELES ${ }^{\mathbf{2}}$; Fábio Denilson de \\ Oliveira FELICIANO ${ }^{3}$; Gil Dutra FURTADO ${ }^{4}$
}

\begin{abstract}
${ }^{1}$ Secteur de la Santé, Cours de Médecine Vétérinaire/Centro Universitário CESMAC, Marechal Deodoro, État de Alagoas, Brésil. E-mail: felicianojr@yahoo.com.br

${ }^{2}$ Médecine Vétérinaire/Universidade Federal de Campina Grande, Brésil (UFCG); Spécialiste des Maladies Infectieuses-Contagieuses des Animaux Domestiques/Universidade Federal Rural de Pernambuco, Brésil (UFRPE); Master en Bioscience Animale/UFRPE; Docteur en Sciences Vétérinaires/UFRPE; Coordinateur et Conférencier du Cours de Médecine Vétérinaire/Centro Universitário Maurício de Nassau, João Pessoa, Brésil (UNINASSAU).E-mail: teles.jaa@gmail.com

${ }^{3}$ Doctorant en Informatique/Universidade Federal de Pernambuco, Brésil (UFPE); Master en Informatique/UFPE; Spécialiste en Gestion de l'Éducation/Serviço Nacional de Aprendizagem Comercial, Brásil (SENAC); Licence en Informatique/Universidade Católica de Pernambuco, Brésil (UNICAP); Professeur Titulaire/Instituto Federal de Educação, Ciência e Tecnologia de Pernambuco, Brésil (IFPE-Campus Belo Jardim). E-mail: fabio.feliciano@belojardim.ifpe.edu.br

${ }^{4}$ Diplômé en Médecine Vétérinaire/UNINASSAU; Agronome/Universidade Federal da Paraíba (UFPB); Doctorat en Psychobiologie/Universidade Federal do Rio Grande do Norte (UFRN); Ingénieur Agronome Partenaire de la Coopérative Agroalimentaire (COOPAGRO). E-mail: gdfurtado@hotmail.com
\end{abstract}

Résumé. La leucose bovine enzootique (LEB) est une maladie infectieuse-contagieuse chronique qui affecte principalement le système lymphoïde du bétail infecté et détermine les processus de désorganisation des tissus et des organes, plus particulièrement les ganglions lymphatiques. L'objectif de cette étude était de déterminer la séroprévalence de LEB dans le troupeau laitier du Sertão de Alagoas. $\mathrm{Au}$ total, 719 échantillons de sérum sanguin provenant de 37 exploitations réparties dans les 26 municipalités du Sertão Alagoano ont été évalués. Les échantillons ont été évalués par rapport au test d'immunodiffusion sur gel d'agar (IDGA), dont 85 (11,82\%) étaient positifs et 634 (88,17\%) étaient négatifs. LEB est répandu dans les troupeaux examinés. Les mesures préventives et sanitaires doivent être mises en œuvre au moyen de tests sérologiques et d'un suivi médical vétérinaire constant visant à assurer l'assainissement progressif des troupeaux.

Mots-clés: Leucémie bovine; Lymphosarcome; Tissus rétrobulbaires; VLB.

\section{SOROPREVALÊNCIA PARA LEUCOSE ENZOÓTICA BOVINA NO REBANHO LEITEIRO DO SERTÃO DO ESTADO DE ALAGOAS, NORDESTE DO BRASIL}


Resumo. A leucose bovina enzoótica (LEB) é uma doença infecciosa contagiosa que afeta principalmente o sistema linfóide de animais infectados e determina os processos de ruptura dos tecidos e órgãos, particularmente dos nódulos linfáticos. O objetivo deste estudo foi determinar a soroprevalência de LEB no rebanho leiteiro de Sertão de Alagoas. Foram avaliadas 719 amostras de soro sanguíneo de 37 fazendas dos 26 municípios do Sertão Alagoano. As amostras foram avaliadas contra o teste de imunodifusão em gel de ágar (IDGA), das quais $85(11,82 \%)$ foram positivas e 634 $(88,17 \%)$ foram negativas. LEB é difundido nos rebanhos examinados. Medidas preventivas e sanitárias devem ser implementadas por meio de testes sorológicos e constante monitoramento médico veterinário, visando garantir saneamento gradual do rebanho.

Palavras-chave: Leucemia bovina; Linfossarcoma; tecido retrobulbar; VLB.

\section{SEROPREVALENCE FOR BOVINE ENZOOTIC LEUKOSIS IN THE DAIRY HERD OF THE 'SERTÃO' FROM STATE OF ALAGOAS, BRAZILIAN NORTHEAST}

Abstract. Enzootic Bovine Leukosis (EBL) is an infectious-contagious chronic disease that primarily affects the lymphoid system of infected livestock and determines the processes of disruption of tissues and organs, particularly the lymph nodes. The objective of this study was to determine the seroprevalence of LEB in the Sertão de Alagoas dairy herd. A total of 719 blood serum samples from 37 farms in the 26 municipalities of Sertão Alagoano were evaluated. Samples were evaluated against the agar gel immunodiffusion test (IDGA), of which 85 (11.82\%) were positive and 634 (88.17\%) were negative. LEB is widespread in the herds examined. Preventive and sanitary measures must be implemented by means of serological tests and constant veterinary medical monitoring aimed at ensuring gradual herd sanitation.

Keywords: Bovine leukemia; Lymphosarcoma; Retrobulbar tissue; VLB.

\section{SEROPREVALENCIA PARA LEUCOSIS BOVINA ENZOÓTICA EN EL REBAÑO LECHERO DEL SERTÓN DEL ESTADO DE ALAGOAS, NORDESTE DEL BRASIL}

Resumen. La leucosis bovina enzoótica (EBL) es una enfermedad crónica infecciosa-contagiosa que afecta principalmente el sistema linfoide del ganado infectado y determina los procesos de ruptura de tejidos y órganos, en particular los ganglios linfáticos. El objetivo de este estudio fue determinar la seroprevalencia de LEB en el hato lechero Sertón de Alagoas. Se evaluaron un total de 719 muestras de suero sanguíneo de 37 granjas en los 26 municipios de Sertón Alagoano. Las muestras se evaluaron frente a la prueba de inmunodifusión en gel de agar (IDGA), de las cuales 85 (11.82\%) fueron positivas y $634(88.17 \%)$ fueron negativas. LEB está muy extendida en los rebaños examinados. Las medidas preventivas y sanitarias deben implementarse mediante pruebas serológicas y un monitoreo médico veterinario constante para garantizar el saneamiento gradual del rebaño.

Palabras clave: Leucemia bovina; Linfosarcoma; Tejido retrobulbar; VLB. 


\section{INTRODUCTION}

La leucose bovine enzootique (LEB) est une maladie chronique infectieuse-contagieuse qui compromet principalement le système lymphoïde du bétail infecté et détermine les processus de désorganisation des ganglions lymphatiques et des tissus. Elle est causée par un virus de la famille des Retroviridae, de la sous-famille Oncovirinae et du genre Deltaretrovirus, qui est par conséquent un virus de l'acide ribonucléique (ARN) du virus. Le virus infecte préférentiellement les lymphocytes, en particulier ceux de type B, bien que l'acide désoxyribonucléique (ADN) pro-viral du virus de la leucose bovine (VLB) ait également été détecté (BARROS FILHO et al., 2009; LEUZZI-JUNIOR; ALFIERI; ALFIERI, 2001; SILVA et al., 2008).

Dans la présente étude, cependant, parmi les troupeaux affectés par le LEB, les bovins laitiers sont les plus touchés en raison de la gestion constante de la production, assurant une transmission iatrogène (horizontale), causée par l'utilisation de motifs tels que l'utilisation de matériel de traite, d'aiguilles, d'instruments chirurgicaux et de la palpation rectale . Par conséquent, les animaux soumis à un régime intensif avec une technologie de pointe sont plus infectés que ceux élevés dans un système extensif (BARROS FILHO et al., 2010).

Les animaux infectés deviennent des porteurs permanents du virus et des transmetteurs potentiels, même sous la forme asymptomatique de la maladie (MEIRELLES et al., 2009). La forme de transmission du virus est liée à l'intégrité des lymphocytes. De cette manière, deux formes de transmission ont été mises en évidence, la forme verticale (passive) et la forme horizontale (active) (SPONCHIADO, 2008).

La transmission verticale du LEB peut se faire par voie transplacentaire ou même par voie orale, par l'ingestion de colostrum et de lait contenant des lymphocytes contaminés par la VLB. La transmission du LEB au cours de la période prénatale, par voie utérine, se produit souvent après le premier trimestre de la gestation chez jusqu'à $8 \%$ des grossesses d'animaux séropositifs. Les vaches à forte concentration en virus et à faible titre en anticorps peuvent transmettre l'infection au fœtus. D'autre part, les femelles à faible concentration en virus et à titre élevé d'anticorps fournissent une immunité passive qui reste pendant quelques mois (SPONCHIADO, 2008).

Dans la forme de transmission horizontale, iatrogène peut être considéré comme très important. Le virus reste viable dans les lymphocytes et la transmission de ceux-ci de manière intégrale (par le sang), du matériel chirurgical, des seringues, des aiguilles, de tatouages et des gants de palpation rectale contaminés peut se produire lorsque les matériaux en question sont 
réutilisés sans précaution. Chez les troupeaux présentant une prévalence élevée de LEB, l'infection iatrogène était l'un des moyens les plus importants (SPONCHIADO, 2008).

La maladie se manifeste sous trois formes distinctes: aleucémique (présence d'anticorps), lymphocytose persistante et développement de lymphosarcomes. De $1 \%$ à $5 \%$ des bovins séropositifs développent un lymphosarcome, la forme la plus courante de néoplasie chez les vaches laitières, et 30\% développent une lymphocytose persistante. L'incidence la plus élevée de tumeurs se situe entre trois et huit ans, avec une mortalité d'environ $10 \%$ à $15 \%$ (AGOTTANI et al., 2012).

Les signes cliniques peuvent inclure perte de poids, augmentation du volume des ganglions lymphatiques due à la prolifération de lymphocytes néoplasiques, diminution de la production laitière, anorexie, incoordination ou paralysie des membres postérieurs par compression de la moelle épinière par des masses néoplasiques, anémie provoquée par une hémorragie au niveau de la moelle épinière. Abomasum, fièvre due à des infections concomitantes, exophtalmie due à une infiltration de lymphocytes néoplasiques dans les tissus rétrobulbaires, dyspnée de compression des voies aériennes due à des ganglions lymphatiques hypertrophiés et insuffisance cardiaque droite (BRAGA et al., 1998; RIET-CORREA et al., 2007).

Les animaux infectés de tous âges développent des anticorps IgM, IgG et IgA qui sont détectés lors de tests sérologiques tels que l'immunodiffusion en gel d'agar (IDGA), la fixation du complément (FC), la séroneutralisation, le dosage radioimmunologique (RIA), le dosage immuno-enzymatique (ELISA) (BRAGA et al., 1998; SPONCHIADO, 2008).

Bien que la leucose enzootique bovine soit une maladie répandue dans le monde entier, le problème n'est pas connu dans l'Etat d'Alagoas. En raison de la rareté des études sur la maladie dans cet État, l'objectif était d'estimer la séroprévalence de la leucose bovine enzootique dans le troupeau laitier de Sertão ‘de Alagoas' (c.-à-d. 'Alagoano').

\section{MATÉRIEL ET MÉTHODES}

Caractérisation de la région. Le Sertão Alagoas est l'une des trois mésorégions appartenant à l'État d'Alagoas, nord-est du Brésil. Il est formé par l'union 26 de municipalités regroupées dans quatre microrégions, à savoir: 'Alagoana do Sertão do São Francisco', 'Batalha', 'Santana do Ipanema' et 'Serrana do Sertão Alagoano'. La mésorégion en question présente un cheptel bovin de 1.221.266 (BRASIL, 2012).

Échantillonnage. Pour composer l'échantillon de cette recherche, la prévalence attendue de la maladie était de $10 \%$, avec un niveau de confiance de $95 \%$ et une erreur 
statistique de $5 \%$, plus $20 \%$ pour pertes (THRUSFIELD, 2004). A la fin, la taille de l'échantillon était de 455 animaux. Cependant, pour cette étude, 719 échantillons de bovins adultes ont été obtenus, répartis sur 37 (trente-sept) propriétés de l'Alagoano sertão. Ces animaux ont été soumis à la pratique d'une gestion similaire, en Régime semi-intensif, avec une rotation intense des animaux.

Collecte d'échantillons. Des échantillons de sang ont été prélevés en juin 2014 par ponction de la veine jugulaire d'animaux âgés de moins de 12 mois ou de veines coccygiennes d'animaux âgés de plus de 12 mois, à l'aide d'aiguilles de 40×12mm. Le sang a été conservé dans des éprouvettes de polypropylène avec une capacité de $10 \mathrm{~mL}$ et le volume de sang recueilli par animal était $( \pm 5 \mathrm{ml})$. Les tubes ont été maintenus à la température ambiante et inclinés pour faciliter la rétraction du caillot et, par conséquent, obtenir du sérum pour les tests sérologiques.

Évaluation sérologique. Les examens ont été effectués dans le laboratoire des maladies infectieuses et contagieuses de l'école de médecine vétérinaire du CESMAC, situé à Marechal Deodoro-AL. La méthode utilisée pour détecter la présence d'anticorps anti-virus LEB était le test d'immunodiffusion sur gel d'agar utilisant le kit commercialisé par l'Institut technologique du Paraná (TECPAR), conformément aux normes du fabricant.

\section{RÉSULTATS}

Sur les 719 échantillons testés par rapport au test d'immunodiffusion sur gel d'agar, 85 (11,82\%) étaient positifs pour le virus de la leucose bovine enzootique et $634(88,17 \%)$ étaient négatifs voir (Tab. 1).

Tableau 1. Résultats des bovins laitiers soumis au test d'immunodiffusion sur gel d'agar (IDGA) contre la leucose bovine enzootique, réalisés dans le Sertão Alagoano Maréchal Deodoro, 2014

\begin{tabular}{cccccc}
\hline Tests & \multicolumn{3}{c}{ Idga* $^{*}$} & Total des échantillons \\
\hline \multirow{2}{*}{$\mathrm{N}^{\mathrm{o}}$ D'échantillons } & Positif & $\%$ & Négatif & $\%$ & 719 \\
\cline { 2 - 5 } & 85 & 11,82 & 634 & 88,17 & \\
\hline
\end{tabular}

*Immunodiffusion en gélose

\section{DISCUSSION}

Considérant que des études menées dans le Nord-est du Brésil par MELO (1991), SILVA (2001), MENDES (2002) et BATISTA (2013) ont montré que la prévalence du LEB était estimée entre $5,1 \%$ et $16,9 \%$. Les résultats trouvés dans cette recherche démontrent une forte corrélation avec eux, renforçant le fait que la réalité de Sertão Alagoano est très proche de celle des autres États du Nord-Est. 
Les études de BIRGEL et al. (1999), évaluant la prévalence du LEB dans le bassin laitier de l'État d'Alagoas, ont montré une prévalence de 9,60\%. En comparant les résultats de cette étude, nous avons observé une légère augmentation du nombre d'animaux infectés par le LEB au fil des ans. Il est suggéré que cette augmentation est due à la négligence de certains créateurs lors de l'acquisition de nouveaux animaux pour composer le troupeau.

Dans l'État de Bahia, une prévalence de 16,1\% a été constatée, dans le Ceará, 9,1\%, dans le Paraíba, 8,3\%, et dans le Sergipe (Est, Agreste et Sertão) avec 11,92\%, ce qui corrobore la prévalence observée dans cette étude; différemment, de Rio Grande do Norte, qui présentait une prévalence de 5,1\% pour le LEB (ABREU; ARAÚJO; BIRGEL, 1994; BATISTA, 2013; SIMÕES, 1998, SIMÕES et al., 2001; TÁVORA et al., 1991). Dans le Maranhão (SANTOS, 2010), on a constaté une prévalence de 53,8\%, ce qui est associé à la commercialisation intensive des animaux dans le but d'améliorer génétiquement les troupeaux de bovins de la laiterie de l'État.

À Pernambuco, des études successives de séroprévalence chez LEB ont confirmé l'existence d'une infection dans différentes mésorégions de cet état: 15,1\% (MELO, 1991), 14,7\% (MENDES, 2002) et 20,7\% (SANTOS et al. , 2013), montrant un pourcentage plus élevé d'animaux affectés par rapport a cette étude.

Dans la région du Nord, les États d'Acre avec une prévalence de 9,7\% pour le LEB et d'Amazonas avec 9,6\% (CARNEIRO et al., 2003; ABREU et al., 1990) ont présenté des résultats similaires à ceux obtenus dans cette étude; tandis que dans la même région, les états de Rondônia et de Tocantins présentaient une prévalence de $23 \%$ et $37 \%$, respectivement, avec des valeurs supérieures à celles trouvées ici (FERNANDES et al., 2009). D'autre part, MOLNÁR et al. (1999) ont analysé 668 échantillons provenant de bovins de différents groupes d'âge appartenant à 14 troupeaux différents, tous dotés d'un système de reproduction étendu et répartis dans différentes microrégions de l'État de Pará, ont révélé une prévalence de $26 \%$. Troupeaux infectés entre $5,4 \%$ et $49 \%$, avec une prédominance de bovins avec un système d'élevage extensif.

Dans la région centre-ouest, l'état de Goiás présentait une prévalence de 35,9\% dans le Mato Grosso do Sul, le taux de prévalence était de $22 \%$, ce qui était proche de la moyenne nationale (ANDRADE; ALMEIDA, 1991).

Des enquêtes ont montré que dans le sud-est du pays, l'état de São Paulo avait un taux de prévalence compris entre $49,2 \%$ et $54 \%$. À Rio de Janeiro, la prévalence était de $54,3 \%$, soit la prévalence la plus élevée observée parmi les études nationales. Dans l'état de Minas Gerais, 
la valeur trouvée était de 28,4\% (BIRGEL et al., 1988; BIRGEL JÚNIOR et al., 1995; D’ANGELINO; GARCIA; BIRGEL, 1998; MEGID et al., 2003; ROMERO et al., 1981).

Dans la région sud du Brésil, le Paraná a une prévalence de 20,7\%, le Rio Grande do Sul de $23,5 \%$ et la prévalence de Santa Catarina était compris entre 7,6\% et $35 \%$, la première valeur étant l'indice le plus bas observé (CORDEIRO et al., 1994; FLORES; WEIBLEIN; REBELATTO, 1990; LUDERS, 2001; POLLETO et al., 2004).

Certaines variables liées au système de sélection, aux formes de gestion hygiénosanitaire adoptées dans les divers biens et états étudiés, ainsi que les méthodes de diagnostic utilisées, les types de race et les technologies utilisées peuvent justifier la variation vérifiée des résultats trouvés (BIRGEL JÚNIOR et al. 1995).

\section{CONCLUSIONS}

La leucose bovine enzootique est répandue dans le troupeau bovin du Sertão Alagoano. Des mesures préventives et sanitaires doivent être mises en place en vue de l'assainissement progressif des troupeaux. Ces mesures devraient reposer sur des tests sérologiques et une surveillance constante de la médecine vétérinaire.

Des études complémentaires doivent être menées dans le but d'établir les facteurs de risque de cette maladie dans le troupeau de l'État.

\section{RÉFÉRENCES}

ABREU, J.M.G.; ARAÚJO, W.P.; BIRGEL, E.H. Prevalência de anticorpos séricos anti- Vírus da Leucose Bovina em animais criados na Bacia Leiteira de Fortaleza, Estado do Ceará. Arquivos da Escola de Medicina Veterinária da Universidade Federal da Bahia, v. 17, p. 67-89, 1994.

ABREU, V.L.V.; MODENA, C.M.; SILVA, J.A.; MOREIRA, E.C.; FIGUEIREDO, M.M.N. Prevalência da Leucose Enzoótica Bovina nos Estados de Rondônia e Acre. Arquivo Brasileiro de Medicina Veterinária e Zootecnia, v. 42, n. 3, p. 203-210, 1990.

AGOTTANI, J.V.B.; OLIVEIRA, K.B.; FAYZANO, L.; WARTH, J.F.G. Leucose Enzoótica Bovina: Diagnóstico, Prevenção e Controle. VP - Laboratório de análises LTDA. Veterinária Preventiva. Curitiba-PR. 2012.

ANDRADE, J.R.A.; ALMEIDA, M.M.R. Prevalência da Leucose Enzoótica Bovina na bacia 
leiteira de Goiânia, Goiás. A Hora Veterinária v. 60, p. 49-53, 1991.

BARROS FILHO, I.R.; GUIMARÃES, A.K.; SPONCHIADO, D.; KRÜGER, E.R.; WAMMES, E.V.; OLLHOFF, R.D.; DORNBUSCH, P.T.; BIONDO, A.W. Soroprevalência de anticorpos para o vírus da leucose enzoótica em bovinos criados na região metropolitana de Curitiba, Paraná. Arquivos do Instituto Biológico, v. 77, n. 3, p. 511-515, jul./set., 2010.

BARROS FILHO, I.R.; GUIMARÃES, A.K.; BIONDO, A.W.; KRÜGER, E.R.; WAMMES, E.V.; OLLHOFF, R.D.; PIEKARZ, C.H.; SPONCHIADO, D. Prevalência da leucose enzoótica em bovinos leiteiros criados na região metropolitana de Curitiba - Paraná. Ciência Animal Brasileira, Suplemento I, Anais do VIII Congresso Brasileiro de Buiatria, p. 513-518, 2009.

BATISTA, J.M. Prevalência de anticorpos séricos anti-vírus da leucose enzoótica bovina em rebanhos bovinos do estado de Sergipe. Universidade Federal da Bahia. Escola de Medicina Veterinária e Zootecnia. Programa de pós-graduação em Ciência Animal nos Trópicos. Salvador-BA, 2013.

BIRGEL, E.H.; AYRES, M.C.C.; BIRGEL JÚNIOR, E.H. Prevalência de anticorpos séricos anti-vírus da leucose enzoótica dos bovinos, em animais criados na bacia leiteira do estado de Alagoas, Brasil. Congresso Brasileiro de Buiatria, 3, Arquivos do Instituto Biológico, São Paulo, v. 129, 1999.

BIRGEL, E.H.; D’ANGELINO, J.L.; GARCIA, M.; MARÇAL, W.S. Estudo preliminar sobre ocorrência da leucose dos bovinos adultos criados na região de Campinas. In: Conferência Anual da Sociedade Paulista da Medicina Veterinária, v. 43, Campinas-SP. Resumos. Campinas: SPMV: 1988. p. 30.

BIRGEL JÚNIOR, E.H.; D’ANGELINO, J.; BENESI, F.J.; BIRGEL, E.H. Prevalência da Infecção pelo vírus da Leucose dos Bovinos em Animais da Raça Jersey, Criados no Estado de São Paulo. Pesquisa Veterinária Brasileira, v. 15, n. 4, p. 93-99, 1995.

BRAGA, F.M.; LANN, C.W. V.D.; SCHUCH, L.F.; HALFEN, D.C.; Infecção Pelo Vírus da Leucose Enzoótica Bovina (BLV). Ciência Rural, Santa Maria, v. 28, n. 1, p. 163-172, 1998. 
BRASIL. Instituto Brasileiro de Geografia e Estatística - IBGE. Produção Pecuária Municipal - PPM. Brasília (DF); 2012.

CARNEIRO, P.A.M.; ARAÚJO, P.W.; BIRGEL, E.H.; SOUSA, K.W. Prevalência da infecção pelo vírus da leucose dos bovinos em rebanhos leiteiros criados no Estado do Amazonas. Acta Amazônica, v. 33, p.111-125, 2003.

CORDEIRO, J.L.F.; DESCHAMPS, F.C.; MARTINS, E. MARTINS, V.M.V. Identificação e controle da leucose enzoótica bovina (LEB) em um rebanho leiteiro. Pesquisa Agropecuária Brasileira, v. 29, n. 8, p. 1287-1292, 1994.

D’ANGELINO, J.L.; GARCIA, M.; BIRGEL, E.H. Epidemiological study of enzootic bovine leukosis in Brazil. Tropical Animal Health and Production, Dordrecht, v. 30, p. 13-15, 1998.

FERNANDES, C.H.C.; MELO, L.G.H.; TENÓRIO, T.G.S.; MENDES, E.J.; FERNANDES, A.C.C.; RAMALHO, T.R.R.; SOBRINHO, P.A.M.; MOTA, R.A. Soroprevalência e fatores de risco da infecção pelo vírus da leucose dos bovinos em rebanhos leiteiros da região norte do estado do Tocantins, Brasil. Arquivos do Instituto Biológico, n. 76, p. 327-334, 2009.

FLORES, E.F.; WEIBLEIN, R.; REBELATTO, M.C. Aspectos epidemiológicos da infecção pelo vírus da leucose enzoótica bovina (BLV) na região central do Rio Grande do Sul, Brasil. A Hora Veterinária, Porto Alegre, v. 10, n. 58, p.25-29, 1990.

LEUZZI-JUNIOR, L.A.; ALFIERI, A.; ALFIERI, A.A. Leucose enzoótica bovina e vírus da leucemia bovina. Semina: Ci. Agrárias, Londrina, v. 22, n.2, p. 211-221, 2001.

LUDERS, M.A. Prevalência de anticorpos contra o vírus da Leucose Enzoótica Bovina em fêmeas com mais de dois anos no rebanho de Bovinos leiteiros no município de Mafra-SC. 2001. (Monografia) - Universidade do Estado de Santa Catarina.

MEGID, J.; NOZAKI, C.N.; KURODA, R.B.S.; CRUZ, T.F.; LIMA, K.C. Ocorrência de leucose enzoótica bovina na microrregião da Serra de Botucatu. Arquivo Brasileiro de Medicina Veterinária e Zootecnia, Belo Horizonte, v. 55, n. 5, p. 645-646, 2003. 
MEIRELlES, C.; DITTRICH, T.; CIPRIANO, F.; OLlHOFF, R.D. Evolução da soroprevalência da leucose enzoótica bovina em um rebanho bovino leiteiro universitário. Ciências agrárias, Londrina. p. 671-678, 2009.

MELO, L.E.H. Leucose Enzoótica dos Bovinos. Prevalência da infecção em rebanhos leiteiros criados no Agreste Meridional do Estado de Pernambuco. 1991. 102 f. (Dissertação de Mestrado) - Faculdade de Medicina Veterinária e Zootecnia, Universidade de São Paulo, São Paulo.

MENDES, E.I. Aspectos Sorológicos e Hematológicos como recursos auxiliares ao diagnóstico da Leucose Enzoótica dos Bovinos em rebanhos leiteiros de Pernambuco. 2002. 47 f. (Dissertação de Mestrado) - Universidade Federal Rural de Pernambuco, Recife.

MOLNÁR, E.; MOLNÁR, L.; DIAS, H.T.; SILVA, A.O.A.; VALE, W.G. Ocorrência da Leucose Enzoótica dos Bovinos no Estado do Pará, Brasil. Pesquisa Veterinária Brasileira, v.19, n.1, p.7-11, 1999.

POLLETO, R.; KREUTZ, L.C.; GONZALES, J.C.; BARCELLOS, L.J.G. Prevalência de tuberculose, brucelose e infecções víricas em bovinos leiteiros do município de Passo Fundo, RS. Ciência Rural, Santa Maria, v. 34, n.2, p. 595-598, 2004.

RIET-CORREA, F.; SCHILD, A. L.; LEMOS, R. A. A.; BORGES, J. R. J. Doenças de ruminantes e eqüídeos. $3^{\mathrm{a}}$ Edição. Santa Maria: Palloti, v.01, 2007. 722 f.

ROMERO, C.H.; ROWE, C.A. Enzootic bovine leukosis virus in Brazil. Tropical Animal Health and Production, Dordrech, v. 13, n. 2, p. 107-111, 1981.

SANTOS, G.R.; OLIVEIRA, J.M.B.; BRANDESPIM, D.F.; OLIVEIRA, A.A.F.; MOTA, R.A.; PINHEIRO JÚNIOR, J. W. Análise epidemiológica da infecção pelo vírus da leucose enzoótica bovina (LEB), na microrregião Garanhuns, Pernambuco, BRASIL. Revista Brasileira de Medicina Veterinária, v. 35, n. 4, p. 371-377, out/dez 2013.

SANTOS, H.P. Leucose Enzoótica Bovina: estudo epidemiológico na bacia leiteira no Estado do Maranhão e aperfeiçoamento do diagnóstico. 2010. 87 f. (Tese de Doutorado) - 
Universidade Federal Rural de Pernambuco, Recife.

SILVA, R.C.; FONTANA, I.; MEIRELLES, F.C.; RUGGIERO, A.P.M.; BENATO, N.; BORGES, J. R. J. Ocorrência de leucoseenzoótica bovina na forma de linfossarcomas no Distrito Federal: relato de caso. Arquivos do Instituto Biológico, São Paulo, v. 75, n. 4, p. $507-$ 512, out./dez., 2008.

SILVA, S.V. Leucose Enzoótica dos Bovinos: Prevalência de anticorpos séricos anti-Vírus da Leucose dos Bovinos em rebanhos cruzados -holandês/zebu e em animais da raça Péduro, criados no Estado do Piauí. 2001. 176 f. (Tese de Doutorado) - Faculdade de Medicina Veterinária e Zootecnia, Universidade de São Paulo, São Paulo.

SIMÕES, S.V.D.; BIRGEL, E.H.; BIRGEL JUNIOR, E.H., AYRES, M.A.C. Prevalência da leucose bovina em animais criados no estado do Rio Grande do Norte. In: CONGRESSO BRASILEIRO DE BUIATRIA, 4., 2001. Campo Grande. Anais... Campo Grande, 2001.

SIMÕES, S.V.D. Leucose Enzoótica dos Bovinos. Prevalência de anticorpos séricos antivírus da Leucose dos Bovinos em rebanhos leiteiros criados no Estado da Paraíba. 1998. 118 f. (Dissertação de Mestrado), Faculdade de Medicina Veterinária e Zootecnia, Universidade de São Paulo, São Paulo.

SPONCHIADO, D. Prevalência de anticorpos séricos anti-vírus da leucose enzoótica bovina em rebanhos da raça Holandesa Preta e Branca, criados no estado do Paraná. 2008. 101 f. (Dissertação de Mestrado em Ciências Veterinárias) - Universidade Federal do Paraná, Curitiba.

TÁVORA, J.P.F.; BIRGEL, E.H. Prevalência da infecção pelo vírus da leucose bovina em rebanhos leiteiros criados na região de Pólo Itabuna, Estado da Bahia. Arquivo da Escola de Medicina Veterinária da Universidade Federal da Bahia, Salvador, v.14, n.1, p.164-83, 1991.

THRUSFIELD, M.V. Epdemiologia veterinaria. 2a Edição. São Paulo: Roca, 2004. 556 f. 